\title{
Systematic Analysis of Environmental Issues on Ecological Smart Bee Farm by Linear Regression Model
}

\author{
A.B.M. Salman Rahman ${ }^{1}$, Myeongbae Lee ${ }^{2}$, Jonghyun $\mathrm{Lim}^{3}$, Yongyun $\mathrm{Cho}^{4}$, and \\ Changsun $\operatorname{Shin}^{5^{*}}$ \\ Department of Information \& Communication Engineering, Sunchon National \\ University, South Korea \\ ${ }^{1}$ salman01717@gmail.com, ${ }^{2}$ lmb@scnu.ac.kr, ${ }^{3}$ sshb56@s.scnu.ac.kr, \\ 4yycho@sunchon.ac.kr, ${ }^{5 *}$ csshin@sunchon.ac.kr
}

\begin{abstract}
Environmental food and nutritional protection primarily depend on pollination from bees. Historically, beekeeping has been performed in different locations as part of the local food community. Beekeeping is increasing rapidly these days due to the high demand for honey and farmers are taking various forms of beekeeping methods to achieve high yield. Honey production also depends on different types of environmental factors. The main principle of this study is to show the analysis results of various types of environmental factors for three different bee farms by the linear regression model to figure out the best farm among all three farms. To improve the production of honey, farmers have to consider different types of environmental factors and this is the elevated time to support farmers by technology. This study analyzed different types of environmental factors like farm outside temperature, farm inside temperature, farm humidity for three different smart bee farms by using a linear regression model to know about their environmental conditions. The performance of prediction models is measured by $R^{2}$ error, Root Mean Squared Error (RMSE), Standard Error values (SE), and Mean Absolute Error (MAE). Based on the outcome, it is observed that the best results giving farm is farm 3 that has been able to give $R^{2}$ value 0.95,0.95, and 0.72 for the farm outside temperature, inside temperature, and farm humidity.
\end{abstract}

Keywords: Honey bee, Environmental factor, Farm outside temperature, Farm inside Temperature, Farm humidity, Linear regression

\section{Introduction}

Nowadays, beekeeping is one of the top fields for increasing employment and income for people in the world. The key component of beekeeping is honey and also one of the most popular sweet liquids made by bees using the nectar from flowers. Beekeeping has historically been carried out in different areas as part of the local culture and also as part of the production of pollinators. Beekeeping is so much popular in several countries all over the world. For example, honey is an essential component of food culture in countries such as Korea, Japan, Bangladesh, India, etc. [1]. Beekeeping and the processing of honey can also

Article history:

Received (October 18, 2020), Review Result (December 5, 2020), Accepted (February 2, 2021)

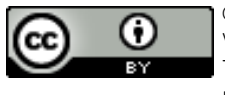

(c) 2021 A.B.M. Salman Rahman, Myeongbae Lee, Jonghyun Lim, Yongyun Cho, and Changsun Shin. Published by Global Vision Press

This is an open access article distributed under the terms of the Creative Commons Attribution License (CC BY4.0), which permits unrestricted use, distribution, and reproduction in any medium, provided the original author and source are 
make a huge contribution to the development of the food community. This is high time to support beekeepers by technology for getting a high profit and technology-based farming called smart farming. Smart farming is an evolving notion that utilizes technology such as IoT, robots, drones, and AI to control farms to increase the quantity and efficiency of goods while optimizing the human labor needed by manufacturing [2].

Beekeeping contributes to the survival of forest societies according to Nongjeonhoiyo [3]. Korea has a long history of beekeeping, more than 2000 years ago beekeeping began in Korea [4]. During the Chosun dynasty (1392-1910), around half of the regions on the Korean peninsula engaged in beekeeping [5]. Exotic bees accounted for $83 \%$ of beehives in the year 2002 in South Korea and now exotic bees are prevalent there. Typically, indigenous species are kept deep in the mountains, while exotic species are kept at the outskirts of mountain areas, including agricultural areas [6][7].

This study focused on the analysis of the environmental issues of smart bee farms using the linear regression model. Related works can be found in section 2, section 3 describes data, methods, and metrics to evaluate the linear regression model, section 4 describes findings and discussion, and finally, section 5 suggested the conclusion of this paper.

\section{Related works}

The survival of an apiculture environment depends on continuous access to high-quality bee forage supplies to maintain stable and productive colonies. Many beekeepers, such as those in Europe and the United States of America, migrate their hives to obtain forage resources after honey flows through public and private property [8]. Environmental relationships among plants and pollinators are strongly affected by Spatio-temporal changes in plant flora and pollinator population dynamics. To understand plant-pollinator linkages in the local climate, the flowering phenology of plant species, and the population patterns of major pollinators, appropriate spatial and temporal gradients should be analyzed [9]. Incompetence rates for honey bee colonies have risen significantly throughout much of North America and Europe, drawing emphasis on the need to better understand the growth and development period of the bee colony, as well as the causes that may cause colony collapse. A basic design of the dynamics of a honey bee colony as a method for exploring which variables have the largest influence on colony development and survival [10].

\section{Materials and methods}

In this study, the researchers used smart bee farm environmental data for every minute of three smart bee farms from March 2020 to April 2020 which is consists of 53826 rows and 6 columns.

The data set is gained from three ecological smart bee farms at Gwangyang is a city in South Jeolla Province, South Korea. In this smart bee farm data, we have "farm outside temperature", "farm inside temperature", and "farm humidity" for three different smart bee farms. For every farm outside temperature, inside temperature, and humidity are also different. [Figure 1] shows the actual values of the outside temperature for every day of three farms in box plots. From the figures, we can see the maximum, minimum, and average line of farms outside temperature. [Figure 2] shows the actual values of inside temperature for every day of three farms in box plots. From the figures, we can see the maximum, minimum, and average line of farms inside temperature. [Figure 3] shows the actual values of the inside humidity of three farms for every day in box plots. From the figures, we can see the maximum, minimum, and average line of farm humidity. 

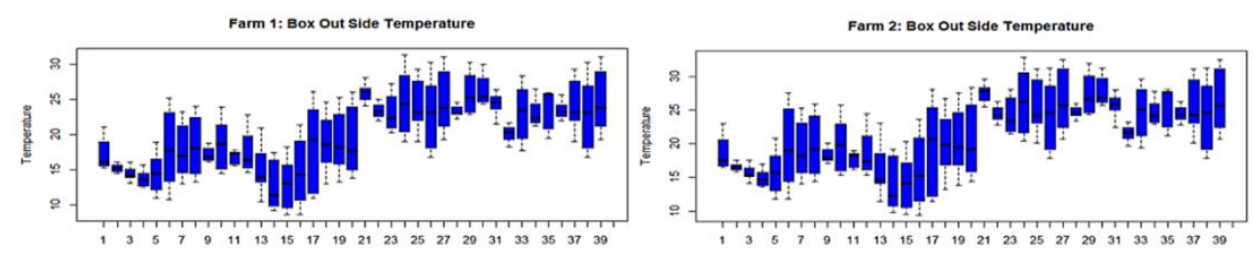

Day

Farm 3: Box Out Side Temperature

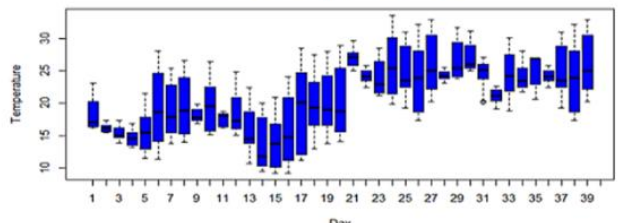

Day

Figure 1. The diagram shows three farms outside temperatures separately for every day
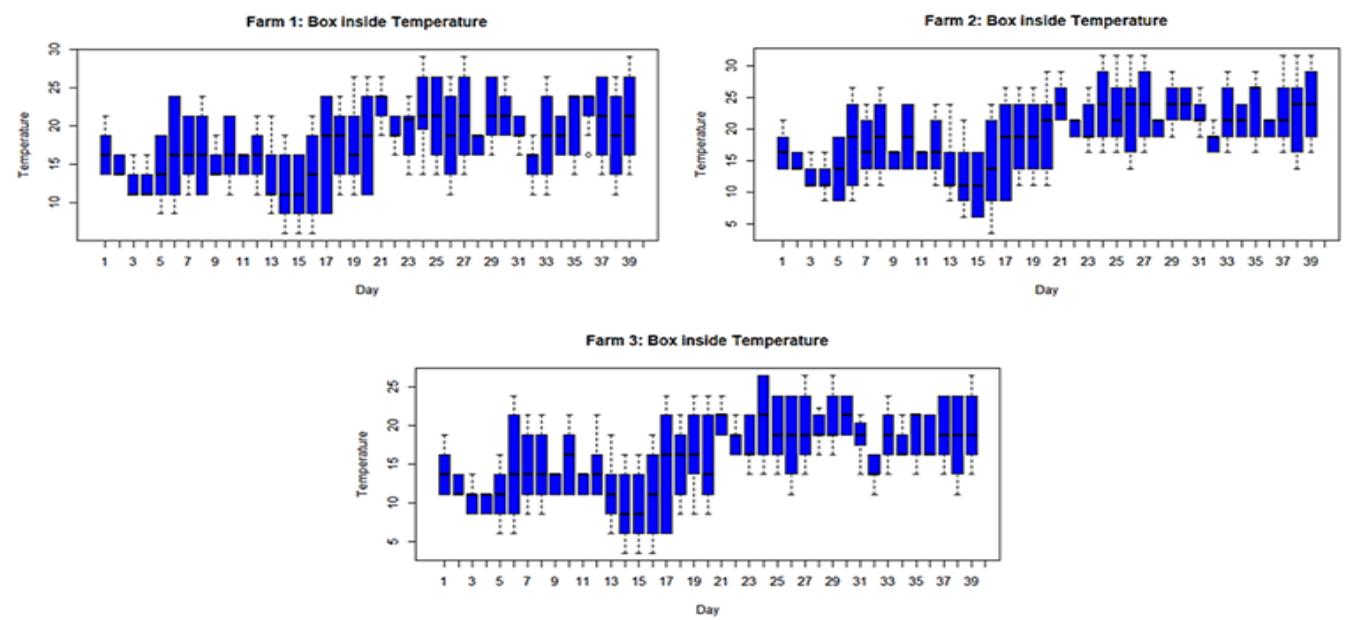

Figure 2. The diagram shows three farms inside temperatures separately for every day

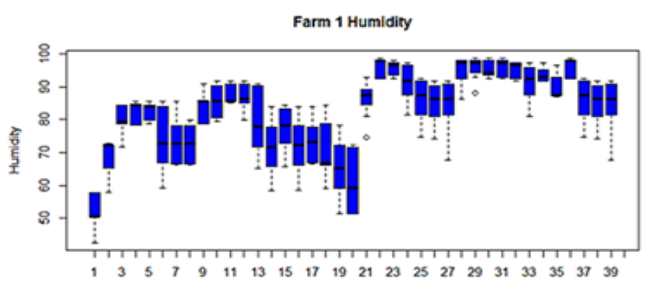

Day

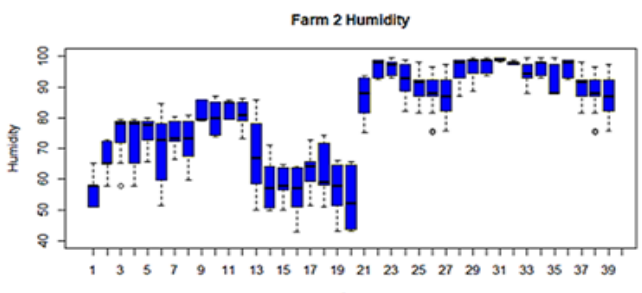

Day

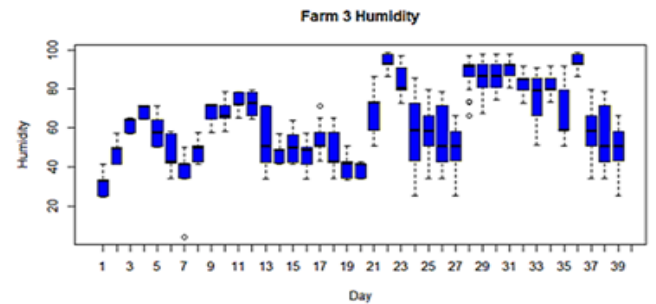

Figure 3. The diagram shows three farms humidity separately for every day 


\subsection{Linear Regression}

A method of modeling to evaluate the relationship between a scalar dependent variable $y$ and one or more independent variables $X$ is called Linear regression. The case of one explanatory variable is known to be a simple linear regression. The approach of two or more explanatory variables is called multiple linear regression [11].

We assume a linear regression, multivariate model,

$$
Y=\beta_{0}+\beta_{1} X+\ldots .+e
$$

In this, $Y$ for the dependent variable, $X$ for the explanatory variable, $\beta_{0}$ and $\beta_{1}$ considered for two unknown constants to represent the intercept and slope, and for error $e$.

\subsection{Performance evaluation indices}

To determine the best prediction results we consider R2 values, Standard Error values (SE), Root Mean Square Error (RMSE), and Mean Absolute Error (MAE). The ranges of R2 values from 0.0 - 1.0 and higher values represent a stronger deal [12]. So, if the R2 value is close to one means it gives good prediction results. In the goodness-of-fit portion of most statistical performance, we consider the standard regression error, also known as the standard estimate error, near R-squared [13]. So, if standard error values are lees and also near to R-squared then the result is good.

The average model prediction error is expressed by both MAE and RMSE in units of the interest variable. The two metrics will vary from 0 to $\infty$ and are oblivious to the error direction. They are negatively driven reviews, which suggests that lower values are good [14].

\section{Result and discussion}

For three smart bee farms, we analyze outside temperature, inside temperature, and humidity in the result portion by using the linear regression model to find the best prediction results among three smart bee farms. [Figure 4] shows the comparison of the actual curve for Farm 1, Farm 2, and Farm 3 outside temperature with the linear regression fitted line separately.
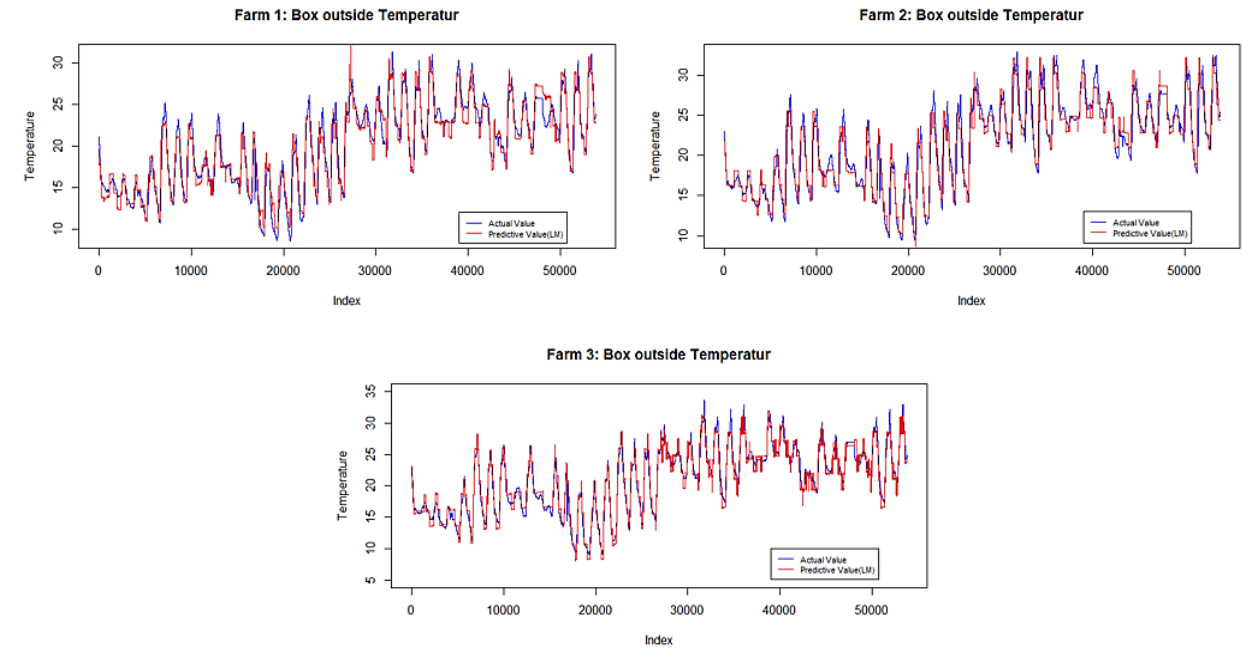

Figure 4. The diagram shows three farms outside the temperature curve with the LM line separately for everyday 
The blue line represents the real value in [Figure 4] and the red line shows the predictive value of the outside temperature for Farm 1, Farm 2, and Farm 3 separately. From the figure, we can see the prediction line very close to the actual line.

[Figure 5] shows the comparison of the actual curve of Farm 1, Farm 2, and Farm 3 inside temperature with the linear regression fitted line separately. The blue line represents the real value in [Figure 5] and the red line shows the predictive value of the inside temperature for Farm 1, Farm 2, and Farm 3 separately. From the figure, we can see the prediction line very close to the actual line. [Figure 6] shows the comparison of the actual curve of Farm 1, Farm 2, and Farm 3 humidity with the linear regression fitted line separately
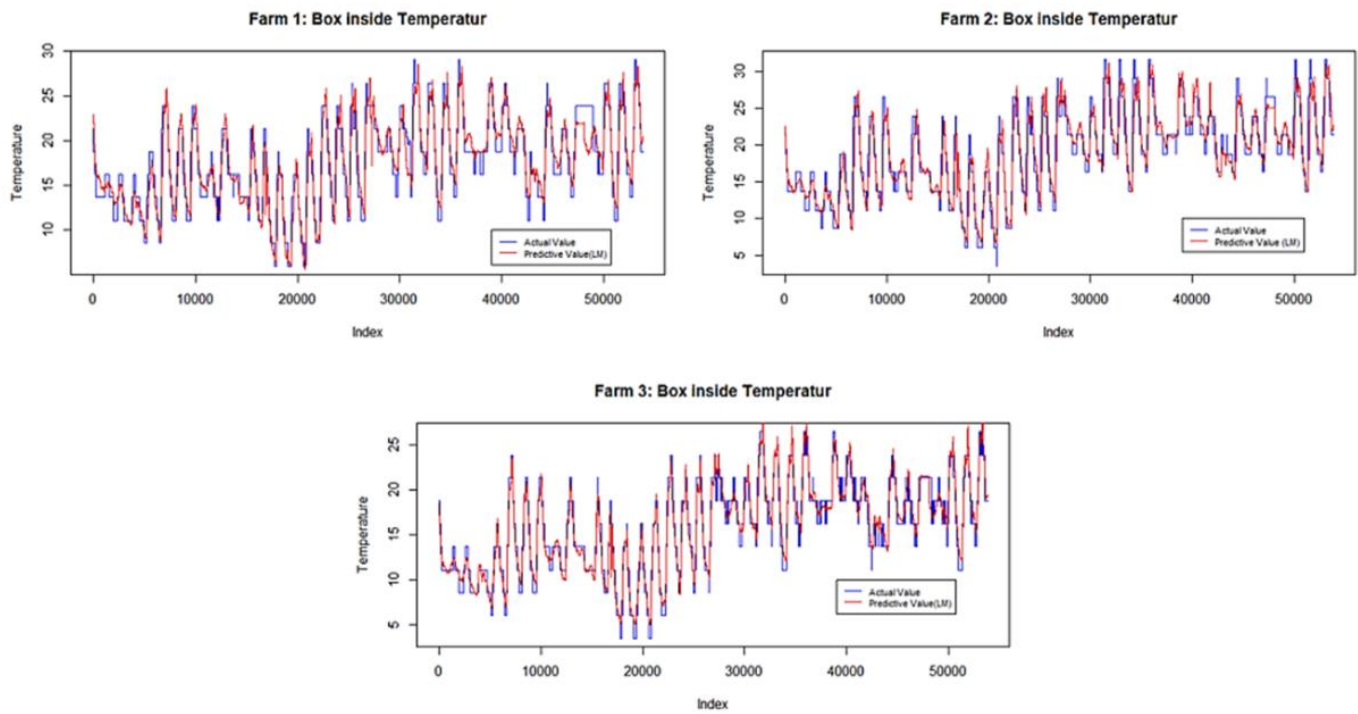

Figure 5. The diagram shows three farms inside the temperature curve with the LM line separately for everyday
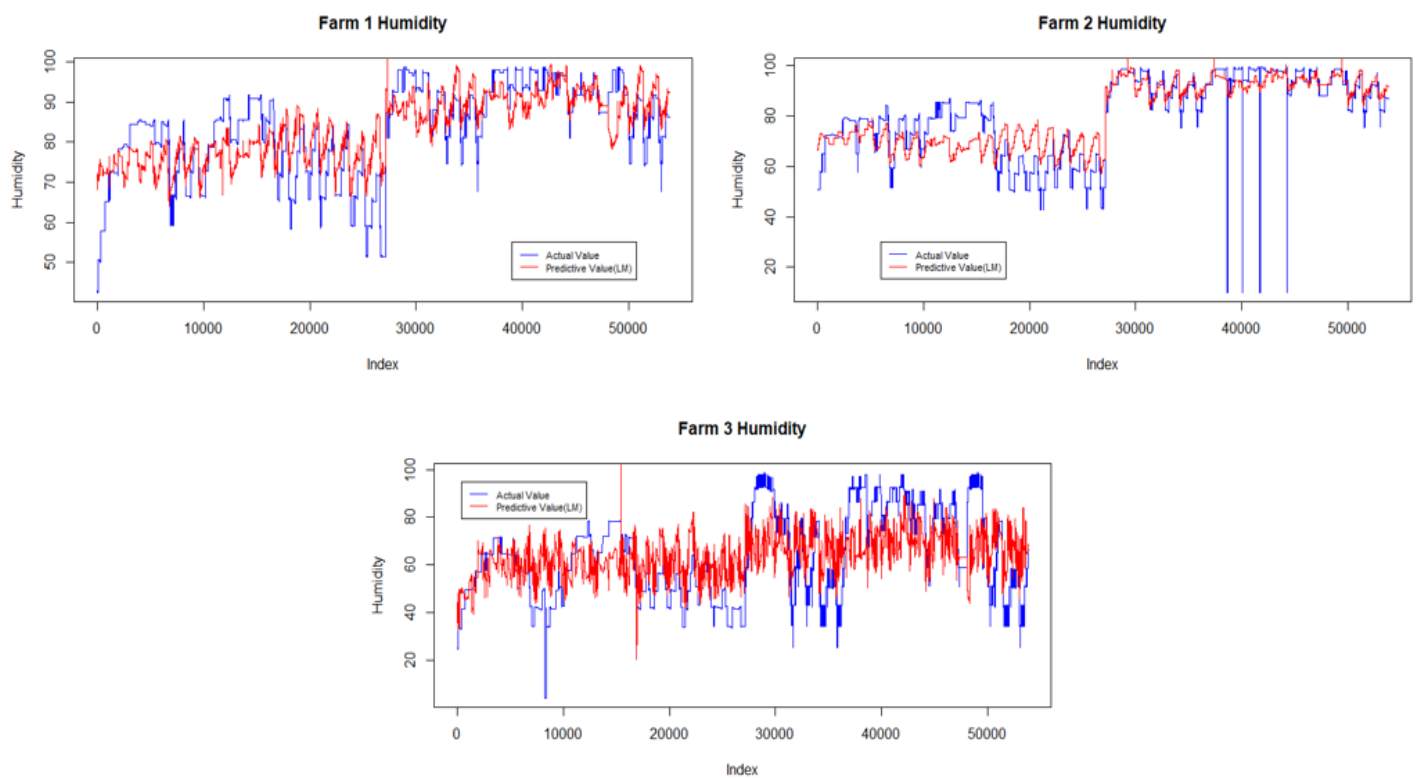

Figure 6. The diagram shows three farms humidity curve with the LM line separately for everyday 
The blue line represents the real value in [Figure 6], and the red line shows the predictive value of humidity for Farm 1, Farm 2, and Farm 3 separately. From the figure, we can see the prediction line very close to the actual line.

In quintessence, all analyses and results are come up with an acuteness for the environmental factors prediction curve. From [Table 1] we can easily find out the best prediction results among three smart bee farms. [Table 1] accumulates all $\mathrm{R}^{2}$ values, Standard Error values (SE), Root Mean Square Error (RMSE), and Mean Absolute Error (MAE) for three smart bee farms outside temperature, inside temperature, and humidity to make a comparison among three smart bee farms.

Table 1. Comparison results for r-square value and standard error value for three smart bee farms

\begin{tabular}{|c|c|c|c|c|c|c|c|c|c|c|c|c|}
\hline \multirow[b]{2}{*}{ Farm } & \multicolumn{4}{|c|}{ Farm Outside Temperature } & \multicolumn{4}{|c|}{ Farm Inside Temperature } & \multicolumn{4}{|c|}{ Farm inside Humidity } \\
\hline & $\begin{array}{c}\text { R- } \\
\text { Squa } \\
\text { re } \\
\text { Valu } \\
\text { e }\end{array}$ & SE & $\begin{array}{c}\text { MA } \\
\text { E }\end{array}$ & RMSE & $\begin{array}{c}\text { R- } \\
\text { Square } \\
\text { Value }\end{array}$ & SE & $\begin{array}{c}\text { MA } \\
\text { E }\end{array}$ & RMSE & $\begin{array}{c}\text { R- } \\
\text { Squar } \\
\mathrm{e} \\
\text { Value }\end{array}$ & SE & $\begin{array}{c}\text { MA } \\
\text { E }\end{array}$ & $\begin{array}{c}\text { RMS } \\
\text { E }\end{array}$ \\
\hline Farm 1 & 0.93 & 1.32 & 1.04 & 1.33 & 0.89 & 1.62 & 1.27 & 1.62 & 0.50 & 7.92 & 6.4 & 8.76 \\
\hline Farm 2 & 0.92 & 1.41 & 1.05 & 1.41 & 0.90 & 1.77 & 1.23 & 1.57 & 0.66 & 8.77 & 6.33 & 7.91 \\
\hline Farm 3 & 0.95 & 1.13 & 0.91 & 1.13 & 0.95 & 1.09 & 0.88 & 1.09 & 0.72 & 6.39 & 6.15 & 7.36 \\
\hline
\end{tabular}

[Table 1] shows us the $\mathrm{R}^{2}$ value and standard error for outside temperature, inside temperature, and also humidity among three smart bee farms by using the Linear Regression Model (LM). From table 1 we can easily find out the best prediction results giving smart bee farms based on environmental factors. After analyzing the outside temperature, inside temperature, and humidity of three smart bee farms we find out the best prediction results giving farm is Farm 3 based on $\mathrm{R}^{2}$ values, RMSE, MAE, and SE. $\mathrm{R}^{2}$ values of Farm 3, for outside temperature, inside temperature, and humidity are $0.95,0.95$, and 0.72 . RMSE values for Farm 3, for outside temperature, inside temperature, and humidity are 1.13, 1.09, and 7.36. RMSE values for Farm 3, for outside temperature, inside temperature, and humidity are 0.91, 0.88 , and 6.15. Standard error values of Farm 3, for outside temperature, inside temperature, and humidity are $1.13,1.09$, and 6.39 .

\section{Conclusions}

The bee has various natural habitats, such as agricultural crop pollination and direct sources of income from the beekeeping industry like honey, bee venom, propolis, pollen, and wax. This study focused on finding out the environmental factor's prediction of three smart bee farms by using the linear regression model. Also, it is intended to find the best smart bee farm among three smart bee farms for getting good prediction results. All results and analysis give us acuteness between environmental factors predictions and smart bee farms. Results are about three different types of environmental factors for three different farms and from the results, we find out that Farm 3 gives us the best result among the three smart bee farms. So, after analyzing all environmental factors we can say Farm 3 is better than the other two farms for getting good production. 


\section{Acknowledgments}

This work has been carried out with the support of "Cooperative Research Program for Agriculture Science \& Technology Development (Project No. PJ01188605)" Rural Development Administration, Republic of Korea and has been assisted by the Korea Institute of Planning and Evaluation for Technology in Food, Agriculture, and Forestry(IPET) through "First-generation animal farm substantiation and industrialization" Program(or Project), funded by the Ministry of Agriculture, Food and Rural Affairs(MAFRA)(119051-01). This work has been carried out with the support of the Korea Institute of Energy Technology Evaluation and Planning (KETEP) and the Ministry of Trade, Industry \&amp; Energy (MOTIE) of the Republic of Korea (20194210100230).

\section{References}

[1] Hae-KyungChung, Hye JeongYang, DayeonShin, and Kyung RhanChung, "Aesthetics of Korean foods: The symbol of Korean culture," vol.3, no.3, pp.178-188, Sept., (2016)

[2] Dieisson Pivoto, Paulo Dabdab Waquilb, EdsonTalamini, Caroline Pauletto Spanhol Finocchio, Vitor FranciscoDalla Corte, and Gianade Vargas Mores, "Scientific development of smart farming technologies and their application in Brazil," Information Processing in Agriculture, vol.5, pp.21-32, Mar., (2018)

[3] M.S. Park, Y.C. Youn, "Traditional knowledge of Korean native beekeeping and sustainable forest management," Forest Policy Econ, vol.15, pp.37-45, Feb., (2012)

[4] Ryo Kohsaka, Mi Sun Park, and Yuta Uchiyama, "Beekeeping and honey production in Japan and South Korea: Past and present,” Journal of Ethnic Foods, vol.4, no.2, pp.72-79, June., (2017)

[5] J.B. Ryu, J.S. Bae, and J.W. Jang, "Honey producing counties in 4 major geographic books during Chosun Dynasty,” Korean J Apic, vol.22, pp.93-100, (2007)

[6] D.H. Oh and S.H. Choi, "Farming status and honey quality of Korean native oriental honeybee (Apis cerana Fab.) in Jiri mountain area," Korean J Apic, vol.19, pp.11-16, (2004)

[7] Chuleui Jung, Sang-kyun Cho, "Relationship between honeybee population and honey production in Korea: A historical trend analysis," Journal of Apiculture, vol.30, pp.7-12, 22 Apr., (2015)

[8] Patel, Vidushi, Eloise Biggs, Natasha Pauli, and Bryan Boruff., "Using a social-ecological system approach to enhance understanding of structural interconnectivities within the beekeeping industry for sustainable decision making," Ecology and Society, vol.25, no.2, (2020)

[9] Naoki Inari, Tsutomu Hiura, Masanori J. Toda and Gaku Kudo, "Pollination linkage between canopy flowering, bumblebee abundance and seed production of understory plants in a cool temperate forest," Journal of Ecology, pp.1534-1543, Nov., (2012)

[10] Stephen Russell, and Andrew B. Barron, David Harris, "Dynamic modeling of honey bee (Apis mellifera) colony growth and failure,” Ecological Modelling, vol.265, pp.158-169, Sept., (2013)

[11] Linear Regression, https://en.wikipedia.org/wiki/Linear_regression, Feb., (2020)

[12] David R. Legates, "Evaluating the use of "goodness-of-fit" measures in hydrologic and hydroclimatic model validation," water resources research, vol.35, no.1, pp.233-241, Jan., (1999)

[13] Wen-Chung and Cheng-Te Chen, "Item parameter recovery, standard error estimates, and fit statistics of the win steps program for the family of Rasch models", educational and psychological measurement, vol.65, no.3, pp.376-405, May., (2015)

[14] Jui-Sheng Chou, and Dac-Khuong Bui, "Modeling heating and cooling loads by artificial intelligence for energy-efficient building design," Energy and Buildings, no.82, pp.437-446, (2014) 


\section{Authors}
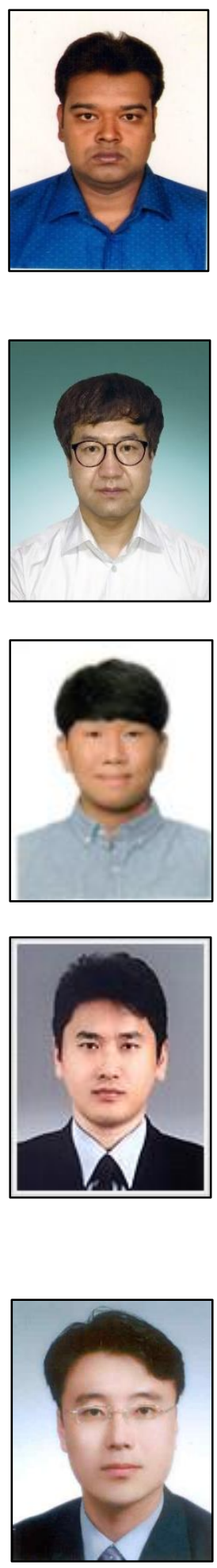

\section{A.B.M. Salman Rahman}

Received the Bachelor's degree from Southeast University, Dhaka, Bangladesh in Electronics and Telecommunication Engineering. At Sunchon National University in South Korea, he is currently pursuing an integrated Ph.D. degree in Information and Communication Engineering. Machine Learning Algorithms, Ubiquitous Computing, and Big Data Processing are his fields of focus.

\section{Myeongbae Lee}

Graduated from Korea with a Bachelor's degree in Computer Engineering. He pursued a Master's degree in Computer Science in South Korea and currently pursuing a Ph.D. degree in Computer and Communication Engineering. His field of focus covers Advanced Agriculture Technology, IT Convergence, Cloud, and Ubiquitous Computing.

\section{Jonghyun Lim}

Graduated from Korea with a Bachelor's degree in Information and Communication Engineering. He is currently studying at Sunchun University for a master's degree in Information and Communication Engineering. System Software and Ubiquitous are among his areas of interest.

\section{Yongyun Cho}

Received the Ph.D. degree from Soongsil University in computer engineering. At present, he is an assistant professor at Sunchon National University in the Department of Information \& communication engineering. System Software, Embedded Software, and Ubiquitous Computing are among his core research interests.

\section{Changsun Shin}

Received the Ph.D. degree from Wonkwang University in Computer Engineering. Presently, he is a professor at Sunchon National University in the Department of Information \& communication engineering. Distributed Computing, IoT, Machine Learning, and Agriculture/ICT Convergence are among his core research interests. 